\title{
Determination of Fifty Trace Element Contents in Macro and Micro Follicular Colloid Nodular Goiter
}

\author{
Vladimir Zaichick ${ }^{1 *}$ and Sofia Zaichick ${ }^{2}$ \\ ${ }^{1}$ Radionuclide Diagnostics Department, Medical Radiological Research Centre, Russia \\ ${ }^{2}$ Feinberg School of Medicine, Northwestern University, USA \\ *Corresponding author: Vladimir Zaichick, Radionuclide Diagnostics Department, Medical Radiological Research Centre, Korolyev \\ St. 4, Obninsk 249036, Kaluga region, Russia.
}

To Cite This Article: Vladimir Zaichick, Sofia Zaichick. Recombinant Determination of Fifty Trace Element Contents in Macro and Micro Follicular Colloid Nodular Goiter. Am J Biomed Sci \& Res. 2021 - 13(6). AJBSR.MS.ID.001931. DOI: 10.34297/AJBSR.2021.13.001931.

Received: 眥 August 01, 2021; Published: 制 August 17, 2021

\begin{abstract}
Background: Colloid nodular goiter (CNG) is the most common disease of the thyroid, even in non-endemic regions, but an etiology of CNG is unclear. It is known that not only iodine (I) but other trace elements (TE) are involved in goitrogenesis. The present study was performed to clarify the preferential accumulation of some TE either in the colloid or in cells of the thyroid gland.

Methods: Fifty TE (Ag, Al, As, Au, B, Be, Bi, Cd, Ce, Co, Cr, Cs, Dy, Er, Eu, Fe, Ga, Gd, Hg, Ho, Ir, La, Li, Lu, Mn, Mo, Nb, Nd, Ni, Pb, Pd, Pr, Pt, Rb, Sb, Sc, Se, Sm, Sn, Tb, Te, Th, Ti, Tl, Tm, U, Y, Yb, Zn, and Zr) in the thyroid tissues with diagnosed CNG were prospectively evaluated in 16 patients with macro-follicular CNG and 13 patients with micro-follicular CNG. Control group included thyroid tissue samples from 105 healthy individuals. Measurements were performed using a combination of non-destructive instrumental neutron activation analysis with inductively coupled plasma mass spectrometry.

Results: It was found that with a goitrous transformation the Ag, Al, B, Bi, Co, Er, Fe, $\mathrm{Hg}, \mathrm{Mo}, \mathrm{Nd}, \mathrm{Ni}, \mathrm{Pb}, \mathrm{Pr}, \mathrm{Rb}, \mathrm{Tl}, \mathrm{U}, \mathrm{Y}, \mathrm{and} \mathrm{Zn}$ level in thyroid tissue can be significantly changed, and these changes depend on CNG histology. An association between $\mathrm{B}$ and Pb content and cell volume of CNG was observed. goiter.

Conclusion: There are considerable changes in TE contents in the goitrous transformed tissue of thyroid, which depend on the histology of

Keywords: Macro and micro follicular colloid nodular goiter of thyroid, Intact thyroid, Trace elements, Instrumental neutron activation analysis, Inductively coupled plasma mass spectrometry

Abbreviations: CNG: Colloid Nodular Goiter; TE: Trace Elements; INAA-LLR: Instrumental Neutron Activation Analysis with high resolution spectrometry of Long-Lived Radionuclides; ICP-MS: Inductively Coupled Plasma Mass Spectrometry; BSS: Biological Synthetic Standards; CRM:Certified Reference Material; IAEA: International Atomic Energy Agency; INCT: Institute of Nuclear Chemistry and Technology; DL: Detection Limit.
\end{abstract}

\section{Introduction}

Colloid nodular goiter (CNG) is the most common disease of the thyroid, even in non-endemic regions [1]. CNG is clinically detected in about $4 \%$ of people older than 30 years [1]. CNG is benign lesion; however, during clinical examination, it can mimic malignant tumors. Furthermore, the origination of CNG can indicate the beginning of malignant transformation of the thyroid gland [2].
Up to now, an etiology of CNG is unclear and probably it is multifactorial [3]. There is opinion that CNG occurs when the thyroid is unable to meet the metabolic demands of the body with sufficient hormone production. The thyroid gland compensates by enlarging, which usually overcomes mild deficiencies of thyroid hormones. For over $20^{\text {th }}$ century, there was the dominant hypothesis that CNG is the simple consequence of iodine (I) deficiency, because 
I is an essential part of thyroid hormones. However, it was found that $\mathrm{CNG}$ is a frequent disease even in those countries and regions where the population is never exposed to I shortage [4]. Moreover, it was shown that I excess has severe consequences on human health and associated with the presence of thyroidal disfunctions and autoimmunity, CNG and diffuse goiter, benign and malignant tumors of gland [5-8]. It was also demonstrated that besides I deficiency and excess many other dietary, environmental, and occupational factors are associated with the CNG incidence [9-11]. Among them a disturbance of evolutionary stable input of many trace elements (TE) in human body after industrial revolution plays a significant role in etiology of thyroidal disorders [12].

Besides I involved in thyroid function, other TE have also essential physiological functions such as maintenance and regulation of cell function, gene regulation, activation or inhibition of enzymatic reactions, and regulation of membrane function [13]. Essential or toxic (goitrogenic, mutagenic, carcinogenic) properties of TE depend on tissue-specific need or tolerance, respectively [13]. Excessive accumulation or an imbalance of the TE may disturb the cell functions and may result in cellular degeneration, death, benign or malignant transformation [13-15].

In our previous studies the complex of in vivo and in vitro nuclear analytical and related methods was developed and used for the investigation of iodine and other TE contents in the normal and pathological thyroid [16-22]. Iodine level in the normal thyroid was investigated in relation to age, gender, and some non-thyroidal diseases $[23,24]$. After that, variations of TE content with age in the thyroid of males and females were studied and age- and genderdependence of some TE was observed [25-41]. Furthermore, a significant difference between some TE contents in normal and cancerous thyroid was demonstrated [42-47].

Histologically, the CNG is cellular hyperplasia of the thyroid acini. There are two histological types of CNG: macro- and microfollicular. It is obvious that these two types of CNG have different volume ratios "colloid to cells".

The present study was performed to clarify the preferential accumulation of some TE either in the colloid or in cells of the thyroid gland. Having this in mind, our aim was to assess the silver (Ag), aluminum ( $\mathrm{Al}$ ), arsenic (As), gold ( $\mathrm{Au}$ ), boron (B),, beryllium $(\mathrm{Be})$, bismuth $(\mathrm{Bi})$, cadmium $(\mathrm{Cd})$, cerium $(\mathrm{Ce})$, cobalt (Co), chromium (Cr), cesium (Cs), dysprosium (Dy), iron (Fe), erbium (Er), europium (Eu), gallium (Ga), gadolinium (Gd), mercury (Hg), holmium (Ho), iridium (Ir), lanthanum (La), lithium (Li), lutecium (Lu), manganese (Mn), molybdenum (Mo), niobium $(\mathrm{Nb})$, neodymium $(\mathrm{Nd})$, nickel $(\mathrm{Ni})$, lead $(\mathrm{Pb})$, palladium $(\mathrm{Pd})$, praseodymium $(\mathrm{Pr})$, platinum $(\mathrm{Pt})$, rubidium $(\mathrm{Rb})$, antimony $(\mathrm{Sb})$, scandium (Sc), selenium (Se), samarium (Sm), tin (Sn), terbium
$(\mathrm{Tb})$, tellurium $(\mathrm{Te})$, thorium (Th), titanium (Ti), thallium ( $\mathrm{Tl}$ ), thulium $(\mathrm{Tm})$, uranium $(\mathrm{U})$, yttrium $(\mathrm{Y})$, ytterbium $(\mathrm{Yb})$, zinc $(\mathrm{Zn})$, and zirconium $(\mathrm{Zr})$ contents in macro- and micro-follicular CNG tissue using consecutively non-destructive instrumental neutron activation analysis with high resolution spectrometry of long-lived radionuclides (INAA-LLR) and inductively coupled plasma mass spectrometry (ICP-MS),. A further aim was to compare the levels of these TE in the macro- and micro-follicular CNG separately with those in intact (normal) gland of apparently healthy persons, as well as to find differences between the levels of these TE in the macro- and micro-follicular CNG.

All studies were approved by the Ethical Committees of the Medical Radiological Research Centre (MRRC), Obninsk. All the procedures performed in studies involving human participants were in accordance with the ethical standards of the institutional and/or national research committee and with the 1964 Helsinki declaration and its later amendments, or with comparable ethical standards.

\section{Material and Methods}

All patients suffered from $C N G(n=29$, mean age $M \pm S D$ was $47 \pm 14$ years, range 30-64) were hospitalized in the Head and Neck Department of the Medical Radiological Research Centre. Thick-needle puncture biopsy of suspicious nodules of the thyroid was performed for every patient, to permit morphological study of thyroid tissue at these sites and to estimate their TE contents. For all patients the diagnosis has been confirmed by clinical and morphological results obtained during studies of biopsy and resected materials. Histological conclusion for all thyroidal lesions was the macro-follicular CNG $(n=16)$ and micro-follicular CNG $(n=13)$.

Normal thyroids for the control group samples were removed at necropsy from 105 deceased (mean age $44 \pm 21$ years, range 2-87), who had died suddenly. Most deaths were due to trauma. A histological examination in the control group was used to control the age norm conformity, as well as to confirm the absence of micro-nodules and latent cancer.

All tissue samples were divided into two portions using a titanium scalpel [48]. One was used for morphological study while the other was intended for TE analysis. After the samples intended for TE analysis were weighed, they were freeze-dried and homogenized [49].

The pounded sample weighing about $10 \mathrm{mg}$ (for biopsy) and 50 $\mathrm{mg}$ (for resected materials) was used for $\mathrm{Ag}$, $\mathrm{Co}, \mathrm{Cr}, \mathrm{Fe}, \mathrm{Hg}, \mathrm{Rb}, \mathrm{Sb}$, Sc, Se, and Zn measurement by INAA-LLR. Details of used nuclear reactions, radionuclides, gamma-energies, spectrometric unit, and sample preparation were presented in our earlier publications 
concerning the INAA-LLR of TE contents in human thyroid, prostate and scalp hair $[29,30,50,51]$.

After non-destructive INAA-LLR investigation the thyroid samples were decomposed in autoclaves and used for ICP-MS. The content of Ag, Al, As, Au, B, Be, Bi, Cd, Ce, Co, Cr, Cs, Dy, Er, Eu, Ga, Gd, $\mathrm{Hg}$, Ho, Ir, La, Li, Lu, Mn, Mo, Nb, Nd, Ni, Pb, Pd, Pr, Pt, Rb, Sb, Se, Sm, $\mathrm{Sn}, \mathrm{Tb}, \mathrm{Te}, \mathrm{Th}, \mathrm{Ti}, \mathrm{Tl}, \mathrm{Tm}, \mathrm{U}, \mathrm{Y}, \mathrm{Yb}, \mathrm{Zn}$, and Zr was determined by ICPMS using an ICP-MS Thermo-Fisher "X-7" Spectrometer (Thermo Electron, USA). The TE concentrations in aqueous solutions were determined by the quantitative method using multi elemental calibration solutions ICP-MS-68A and ICP-AM-6-A produced by High-Purity Standards (Charleston, SC 29423, USA). Indium was used as an internal standard in all measurements. Information detailing with the ICP-MS methods used and other details of the analysis was presented in our previous publication concerning TE contents in human thyroid, prostate, and scalp hair [35,52-55].

To determine contents of the TE by comparison with a known standard, biological synthetic standards (BSS) prepared from phenol-formaldehyde resins were used [56]. In addition to BSS, aliquots of commercial, chemically pure compounds were also used as standards. Ten sub-samples of certified reference material (CRM) of the International Atomic Energy Agency (IAEA) IAEA H-4 (animal muscle) and IAEA HH-1 (human hair), as well as five sub-samples of CRM of the Institute of Nuclear Chemistry and Technology (INCT, Warszawa, Poland) INCT-SBF-4 Soya Bean Flour, INCT-TL-1 Tea Leaves, and INCT-MPH-2 Mixed Polish Herbs were treated and analyzed in the same conditions that thyroid samples to estimate the precision and accuracy of results.

A dedicated computer program for INAA-LLR mode optimization was used [57]. All thyroid samples were prepared in duplicate, and mean values of TE contents were used in final calculation. Using Microsoft Office Excel, a summary of the statistics, including, arithmetic mean, standard deviation, standard error of mean, minimum and maximum values, median, percentiles with 0.025 and 0.975 levels were calculated for TE contents. The difference in the results between normal thyroid and two groups of CNG (separately macro- and micro-follicular), as well as between two groups of CNG was evaluated by the parametric Student's t-test and non-parametric Wilcoxon-Mann-Whitney U-test.

\section{Results}

The comparison of our results for the $\mathrm{Ag}$, $\mathrm{Co}, \mathrm{Cr}, \mathrm{Fe}, \mathrm{Hg}, \mathrm{Rb}, \mathrm{Sb}$, $\mathrm{Se}$, and $\mathrm{Zn}$ mass fractions (mg/kg, dry mass basis) in the normal human thyroid obtained by both INAA-LLR and ICP-MS methods is shown in Table 1.
Table 1: Comparison of the mean values $(\mathrm{M} \pm \mathrm{SEM})$ of the chemical element mass fractions ( $\mathrm{mg} / \mathrm{kg}$, on dry-mass basis) in the normal thyroid of males and females obtained by both NAA-LLR and ICP-MS methods.

\begin{tabular}{|c|c|c|c|}
\hline Element & $\begin{array}{c}\text { NAA-LLR } \\
\text { M1 }\end{array}$ & $\begin{array}{l}\text { ICP-MS } \\
\text { M2 }\end{array}$ & $\Delta, \%$ \\
\hline $\mathrm{Ag}$ & $0.0151 \pm 0.0016$ & $0.0122 \pm 0.0014$ & 19.2 \\
\hline Co & $0.0399 \pm 0.0030$ & $0.0378 \pm 0.0031$ & 5.3 \\
\hline $\mathrm{Cr}$ & $0.539 \pm 0.032$ & $0.451 \pm 0.033$ & 16.3 \\
\hline $\mathrm{Fe}$ & $225 \pm 11$ & $221 \pm 12$ & 1.8 \\
\hline $\mathrm{Hg}$ & $0.0421 \pm 0.0041$ & $0.0794 \pm 0.0114$ & -88.5 \\
\hline $\mathrm{Rb}$ & $7.37 \pm 0.44$ & $7.79 \pm 0.46$ & -5.7 \\
\hline $\mathrm{Sb}$ & $0.111 \pm 0.008$ & $0.079 \pm 0.008$ & 28.8 \\
\hline $\mathrm{Se}$ & $2.32 \pm 0.14$ & $2.12 \pm 0.14$ & 8.6 \\
\hline Zn & $97.8 \pm 4.5$ & $91.8 \pm 4.3$ & 6.1 \\
\hline
\end{tabular}

Tables 2-4 presents certain statistical parameters (arithmetic mean, standard deviation, standard error of mean, minimal and maximal values, median, percentiles with 0.025 and 0.975 levels) of the $\mathrm{Ag}, \mathrm{Al}, \mathrm{As}, \mathrm{Au}, \mathrm{B}, \mathrm{Be}, \mathrm{Bi}, \mathrm{Cd}, \mathrm{Ce}, \mathrm{Co}, \mathrm{Cr}, \mathrm{Cs}, \mathrm{Dy}, \mathrm{Er}, \mathrm{Eu}, \mathrm{Ga}, \mathrm{Gd}$, $\mathrm{Hg}$, Ho, Ir, La, Li, Lu, Mn, Mo, Nb, Nd, Ni, Pb, Pd, Pr, Pt, Rb, Sb, Se, $\mathrm{Sm}, \mathrm{Sn}, \mathrm{Tb}, \mathrm{Te}, \mathrm{Th}, \mathrm{Ti}, \mathrm{Tl}, \mathrm{Tm}, \mathrm{U}, \mathrm{Y}, \mathrm{Yb}, \mathrm{Zn}$, and $\mathrm{Zr}$ mass fraction in normal thyroid $(\mathrm{n}=105)$, macro-follicular CNG $(\mathrm{n}=16)$, and microfollicular CNG ( $\mathrm{n}=13)$, respectively. The $\mathrm{As}, \mathrm{Au}, \mathrm{Eu}, \mathrm{Ho}, \mathrm{Ir}, \mathrm{Lu}, \mathrm{Pd}$, $\mathrm{Pt}, \mathrm{Te}, \mathrm{Th}, \mathrm{Tm}, \mathrm{Yb}$, and $\mathrm{Zr}$ mass fractions in normal thyroid samples were determined in a few samples. The possible upper limit of the mean $(\leq M)$ for these TE was calculated as the average mass fraction, using the value of the detection limit (DL) instead of the individual value when the latter was found to be below the DL: where Ci is the individual value of the TE mass fraction in sample -i, ni is number of samples with mass fraction higher than the DL, nj is number of samples with mass fraction lower than the DL, and $n=n i+n j$ is number of samples that were investigated. The As, Dy, Er, Gd, Ho, Ir, $\mathrm{Lu}, \mathrm{Nb}, \mathrm{Pd}, \mathrm{Pt}, \mathrm{Tb}, \mathrm{Te}, \mathrm{Ti}$, and Tm contents in all samples of goitrous thyroid were under DL.

The comparison of Ag, Al, B, Be, Bi, Cd, Ce, Co, Cr, Cs, Fe, Ga, Hg, La, Li, Mn, Mo, Nd, Ni, Pb, Pr, Rb, Sb, Sc, Se, Sm, Sn, Tl, U, Y, and Zn mass fraction in normal thyroid with those in macro- and microfollicular CNG is shown in Table 5, 6 respectively.

The ratios of means and the difference between mean values of $\mathrm{Ag}, \mathrm{Al}, \mathrm{B}, \mathrm{Be}, \mathrm{Bi}, \mathrm{Cd}, \mathrm{Ce}, \mathrm{Co}, \mathrm{Cr}, \mathrm{Cs}, \mathrm{Fe}, \mathrm{Ga}, \mathrm{Hg}$, La, Li, Mn, Mo, Nd, Ni, Pb, $\mathrm{Pr}, \mathrm{Rb}, \mathrm{Sb}, \mathrm{Sc}, \mathrm{Se}, \mathrm{Sm}, \mathrm{Sn}, \mathrm{Tl}, \mathrm{U}, \mathrm{Y}$, and Zn mass fractions in macro- and micro-follicular CNG are presented in Table 7. 


\begin{tabular}{|c|c|c|c|c|c|c|c|c|}
\hline Element & $\mathbf{M}$ & SD & SEM & Min & Max & Median & P 0.025 & P 0.975 \\
\hline $\mathrm{Ag}$ & 0.0133 & 0.0114 & 0.0013 & 0.0016 & 0.0789 & 0.0102 & 0.00187 & 0.0333 \\
\hline $\mathrm{Al}$ & 10.5 & 13.4 & 1.8 & 0.8 & 69.3 & 6.35 & 1.19 & 52.9 \\
\hline As & $\leq 0.0049$ & - & - & $<0.003$ & 0.02 & - & - & - \\
\hline $\mathrm{Au}$ & $\leq 0.0050$ & - & - & $<0.002$ & 0.0203 & - & - & - \\
\hline B & 0.476 & 0.434 & 0.058 & 0.2 & 2.3 & 0.3 & 0.2 & 1.73 \\
\hline $\mathrm{Be}$ & 0.00052 & 0.0006 & 0.00008 & 0.0001 & 0.0031 & 0.0003 & 0.0001 & 0.0022 \\
\hline $\mathrm{Bi}$ & 0.0072 & 0.0161 & 0.0022 & 0.0003 & 0.1 & 0.0027 & 0.0005 & 0.0523 \\
\hline $\mathrm{Cd}$ & 2.08 & 2.05 & 0.27 & 0.011 & 8.26 & 1.37 & 0.113 & 7.76 \\
\hline $\mathrm{Ce}$ & 0.008 & 0.008 & 0.0011 & 0.001 & 0.0348 & 0.00475 & 0.00134 & 0.0293 \\
\hline Co & 0.039 & 0.0276 & 0.0031 & 0.01 & 0.14 & 0.0285 & 0.013 & 0.124 \\
\hline $\mathrm{Cr}$ & 0.495 & 0.261 & 0.031 & 0.13 & 1.3 & 0.43 & 0.158 & 1.08 \\
\hline Cs & 0.0245 & 0.0166 & 0.0022 & 0.0022 & 0.0924 & 0.0198 & 0.00667 & 0.0723 \\
\hline Dy & 0.00122 & 0.00183 & 0.00025 & 0.0003 & 0.0121 & 0.00063 & 0.0003 & 0.00519 \\
\hline Er & 0.000377 & 0.000367 & 0.00005 & 0.0001 & 0.0022 & 0.000275 & 0.0001 & 0.0011 \\
\hline $\mathrm{Eu}$ & $\leq 0.00039$ & - & - & $<0.0002$ & 0.0019 & - & - & - \\
\hline $\mathrm{Fe}$ & 222.8 & 89.5 & 9.6 & 52 & 474 & 222 & 67.8 & 425 \\
\hline Ga & 0.0316 & 0.0156 & 0.0021 & 0.01 & 0.081 & 0.0295 & 0.01 & 0.07 \\
\hline $\mathrm{Gd}$ & 0.00105 & 0.00109 & 0.00015 & 0.0004 & 0.0065 & 0.0006 & 0.0004 & 0.00425 \\
\hline $\mathrm{Hg}$ & 0.0543 & 0.0373 & 0.0043 & 0.007 & 0.151 & 0.046 & 0.00983 & 0.15 \\
\hline Ho & $\leq 0.00040$ & - & - & $<0.0001$ & 0.0042 & - & - & - \\
\hline Ir & $\leq 00.00028$ & - & - & $<0.0002$ & 0.001 & - & - & - \\
\hline $\mathrm{La}$ & 0.00475 & 0.00461 & 0.00062 & 0.0004 & 0.0219 & 0.0027 & 0.0004 & 0.0171 \\
\hline $\mathrm{Li}$ & 0.0208 & 0.0155 & 0.0022 & 0.0015 & 0.0977 & 0.0178 & 0.00412 & 0.0487 \\
\hline $\mathrm{Lu}$ & $\leq 0.00020$ & - & - & $<0.0001$ & 0.001 & - & - & - \\
\hline $\mathrm{Mn}$ & 1.28 & 0.56 & 0.07 & 0.47 & 4.04 & 1.15 & 0.537 & 2.23 \\
\hline Mo & 0.0836 & 0.047 & 0.0062 & 0.0104 & 0.299 & 0.0776 & 0.0278 & 0.211 \\
\hline $\mathrm{Nb}$ & 0.597 & 0.898 & 0.12 & 0.013 & 3.77 & 0.188 & 0.013 & 3.26 \\
\hline $\mathrm{Nd}$ & 0.0041 & 0.0034 & 0.0004 & 0.0002 & 0.0165 & 0.003 & 0.00064 & 0.0137 \\
\hline $\mathrm{Ni}$ & 0.449 & 0.344 & 0.046 & 0.074 & 1.8 & 0.33 & 0.12 & 1.39 \\
\hline $\mathrm{Pb}$ & 0.233 & 0.246 & 0.033 & 0.023 & 1.6 & 0.18 & 0.0328 & 0.776 \\
\hline $\mathrm{Pd}$ & $\leq 0.022$ & - & - & $<0.014$ & 0.07 & - & - & - \\
\hline $\operatorname{Pr}$ & 0.00107 & 0.00086 & 0.00011 & 0.0001 & 0.0039 & 0.00073 & 0.0002 & 0.0035 \\
\hline $\mathrm{Pt}$ & $\leq 0.00057$ & - & - & $<0.00020$ & 0.0138 & - & - & - \\
\hline $\mathrm{Rb}$ & 7.54 & 3.65 & 0.39 & 1.21 & 22.6 & 6.84 & 3.54 & 17.4 \\
\hline $\mathrm{Sb}$ & 0.0947 & 0.0692 & 0.0075 & 0.0047 & 0.308 & 0.0808 & 0.0117 & 0.279 \\
\hline Sc & 0.0268 & 0.0329 & 0.006 & 0.0002 & 0.086 & 0.0064 & 0.000418 & 0.086 \\
\hline $\mathrm{Se}$ & 2.22 & 1.24 & 0.14 & 0.32 & 5.8 & 1.84 & 0.776 & 5.58 \\
\hline $\mathrm{Sm}$ & 0.000507 & 0.000469 & 0.000064 & 0.0001 & 0.0021 & 0.00035 & 0.0001 & 0.0015 \\
\hline Sn & 0.0777 & 0.0677 & 0.0091 & 0.009 & 0.263 & 0.055 & 0.009 & 0.242 \\
\hline $\mathrm{Tb}$ & 0.000198 & 0.000116 & 0.000016 & 0.00008 & 0.0006 & 0.00015 & 0.0001 & 0.00047 \\
\hline $\mathrm{Te}$ & $\leq 0.0057$ & - & - & $<0.003$ & 0.0185 & - & - & - \\
\hline Th & $\leq 0.0032$ & - & - & $<0.002$ & 0.01 & - & - & - \\
\hline $\mathrm{Ti}^{*}$ & 3.5 & 3.53 & 0.47 & 0.44 & 14.5 & 2.3 & 0.602 & 13 \\
\hline $\mathrm{Tl}$ & 0.000932 & 0.000511 & 0.000068 & 0.0001 & 0.0029 & 0.0009 & 0.000294 & 0.00216 \\
\hline $\mathrm{Tm}$ & $\leq 0.00014$ & - & - & $<0.0001$ & 0.0004 & & & \\
\hline
\end{tabular}




\begin{tabular}{|c|c|c|c|c|c|c|c|c|}
\hline $\mathrm{U}$ & 0.000443 & 0.000434 & 0.000059 & 0.0001 & 0.0026 & 0.0003 & 0.0001 & 0.00131 \\
\hline $\mathrm{Y}$ & 0.0026 & 0.00234 & 0.00032 & 0.001 & 0.011 & 0.0017 & 0.001 & 0.00942 \\
\hline $\mathrm{Yb}$ & $\leq 0.00059$ & - & - & $<0.0003$ & 0.0057 & - & - & - \\
\hline $\mathrm{Zn}$ & 94.8 & 39.6 & 4.2 & 7.1 & 215 & 88.9 & 34.9 & 196 \\
\hline $\mathrm{Zr}$ & $\leq 0.081$ & - & - & $<0.03$ & 0.48 & - & - & - \\
\hline
\end{tabular}

Note*: M - arithmetic mean, SD - standard deviation, SEM - standard error of mean, Min - minimum value, Max - maximum value, P 0.025 percentile with 0.025 level, P 0.975 - percentile with 0.975 level.

\begin{tabular}{|c|c|c|c|c|c|c|c|c|}
\hline Element & $\mathbf{M}$ & SD & SEM & Min & Max & Median & P 0.025 & P 0.975 \\
\hline $\mathrm{Ag}$ & 0.108 & 0.123 & 0.035 & 0.002 & 0.44 & 0.079 & 0.0022 & 0.371 \\
\hline $\mathrm{Al}$ & 26.6 & 25 & 7.2 & 6.6 & 76.3 & 17.5 & 6.77 & 76.2 \\
\hline As & $<0.004$ & - & - & - & - & - & - & - \\
\hline $\mathrm{Au}$ & 0.0115 & 0.0139 & 0.004 & 0.003 & 0.0517 & 0.008 & 0.003 & 0.0432 \\
\hline $\mathrm{B}$ & 1.24 & 0.46 & 0.13 & 0.9 & 2 & 1 & 0.928 & 2 \\
\hline $\mathrm{Be}$ & 0.00055 & 0.00032 & 0.00009 & 0.0002 & 0.001 & 0.0005 & 0.0002 & 0.001 \\
\hline $\mathrm{Bi}$ & 0.056 & 0.066 & 0.019 & 0.0039 & 0.214 & 0.0289 & 0.0065 & 0.2 \\
\hline $\mathrm{Cd}$ & 1.07 & 1.4 & 0.4 & 0.199 & 5.36 & 0.633 & 0.209 & 4.24 \\
\hline $\mathrm{Ce}$ & 0.0189 & 0.0188 & 0.005 & 0.0046 & 0.0696 & 0.0111 & 0.005 & 0.0604 \\
\hline Co & 0.0495 & 0.0254 & 0.0068 & 0.015 & 0.0914 & 0.0509 & 0.0155 & 0.0911 \\
\hline $\mathrm{Cr}$ & 1.39 & 1.73 & 0.45 & 0.255 & 7.3 & 0.976 & 0.282 & 5.54 \\
\hline $\mathrm{Cs}$ & 0.0159 & 0.0068 & 0.002 & 0.0083 & 0.0284 & 0.0161 & 0.0084 & 0.0276 \\
\hline Dy & $<0.005$ & - & - & - & - & - & - & - \\
\hline Er & 0.00167 & 0.00089 & 0.00026 & 0.001 & 0.003 & 0.001 & 0.001 & 0.003 \\
\hline $\mathrm{Eu}$ & $<0.001$ & - & - & - & - & - & - & - \\
\hline $\mathrm{Fe}$ & 431 & 390 & 98 & 65.1 & 1210 & 207 & 72.9 & 1151 \\
\hline $\mathrm{Ga}$ & 0.0203 & 0.0092 & 0.003 & 0.01 & 0.034 & 0.02 & 0.01 & 0.0332 \\
\hline $\mathrm{Gd}$ & $<0.001$ & - & - & - & - & - & - & - \\
\hline $\mathrm{Hg}$ & 1.43 & 1.34 & 0.35 & 0.1 & 5.18 & 1.34 & 0.109 & 4.42 \\
\hline Ho & $<0.0002$ & - & - & - & - & - & - & - \\
\hline Ir & $<0.0003$ & - & - & - & - & - & - & - \\
\hline $\mathrm{La}$ & 0.0097 & 0.0096 & 0.003 & 0.0017 & 0.0356 & 0.00565 & 0.002 & 0.0308 \\
\hline $\mathrm{Li}$ & 0.0268 & 0.0144 & 0.004 & 0.0073 & 0.0541 & 0.0252 & 0.00818 & 0.0535 \\
\hline $\mathrm{Lu}$ & $<0.0002$ & - & - & - & - & - & - & - \\
\hline $\mathrm{Mn}$ & 1.35 & 0.67 & 0.18 & 0.56 & 2.7 & 1.2 & 0.57 & 2.63 \\
\hline Mo & 0.165 & 0.066 & 0.019 & 0.049 & 0.259 & 0.173 & 0.0565 & 0.251 \\
\hline $\mathrm{Nb}$ & $<0.013$ & - & - & - & - & - & - & - \\
\hline $\mathrm{Nd}$ & 0.0131 & 0.0093 & 0.003 & 0.0031 & 0.0331 & 0.0103 & 0.0032 & 0.0312 \\
\hline $\mathrm{Ni}$ & 2.55 & 1.6 & 0.46 & 0.17 & 4.8 & 2 & 0.316 & 4.8 \\
\hline $\mathrm{Pb}$ & 0.383 & 0.201 & 0.058 & 0.12 & 0.74 & 0.38 & 0.12 & 0.721 \\
\hline $\mathrm{Pd}$ & $<0.012$ & - & - & - & - & - & - & - \\
\hline $\operatorname{Pr}$ & 0.00398 & 0.00334 & 0.001 & 0.00068 & 0.0119 & 0.0041 & 0.00071 & 0.0108 \\
\hline $\mathrm{Pt}$ & $<0.0002$ & - & - & - & - & - & - & - \\
\hline $\mathrm{Rb}$ & 9.5 & 4.23 & 0.5 & 2.5 & 22.1 & 9.05 & 3.41 & 19.6 \\
\hline $\mathrm{Sb}$ & 0.0704 & 0.0725 & 0.019 & 0.0102 & 0.267 & 0.041 & 0.0113 & 0.245 \\
\hline Sc & 0.0229 & 0.0407 & 0.011 & 0.0002 & 0.113 & 0.0044 & 0.0002 & 0.112 \\
\hline $\mathrm{Se}$ & 3.45 & 3.17 & 0.82 & 1.29 & 12.6 & 2.24 & 1.29 & 11 \\
\hline $\mathrm{Sm}$ & 0.00149 & 0.00184 & 0.00053 & 0.0004 & 0.0069 & 0.00095 & 0.0004 & 0.0058 \\
\hline
\end{tabular}




\begin{tabular}{|c|c|c|c|c|c|c|c|c|}
\hline $\mathrm{Sn}$ & 0.0467 & 0.0476 & 0.014 & 0.0143 & 0.172 & 0.0274 & 0.0149 & 0.155 \\
\hline $\mathrm{Tb}$ & $<0.0001$ & - & - & - & - & - & - \\
\hline $\mathrm{Te}$ & $<0.007$ & - & - & - & - & - & - \\
\hline $\mathrm{Th}$ & 0.0067 & 0.0057 & 0.002 & 0.002 & 0.0209 & 0.005 & 0.002 & 0.0193 \\
\hline $\mathrm{Ti}^{*}$ & $<0.4$ & - & - & - & - & - & - & - \\
\hline $\mathrm{Tl}$ & 0.00151 & 0.00079 & 0.00023 & 0.00067 & 0.0035 & 0.00135 & 0.00069 & 0.00314 \\
\hline $\mathrm{Tm}$ & $<0.0003$ & - & - & - & - & - & - & - \\
\hline $\mathrm{U}$ & 0.00165 & 0.00052 & 0.00026 & 0.0013 & 0.0024 & 0.00145 & 0.0013 & 0.00234 \\
\hline $\mathrm{Y}$ & 0.0108 & 0.0114 & 0.004 & 0.0036 & 0.0346 & 0.0047 & 0.0036 & 0.0326 \\
\hline $\mathrm{Yb}$ & 0.00024 & 0.00009 & 0.00003 & 0.0002 & 0.0004 & 0.0002 & 0.0002 & 0.0004 \\
\hline $\mathrm{Zn}$ & 132 & 55 & 14 & 84 & 264 & 117 & 86.5 & 261 \\
\hline $\mathrm{Zr}$ & 0.074 & 0.054 & 0.016 & 0.031 & 0.205 & 0.051 & 0.031 & 0.187 \\
\hline $\mathrm{N}$ & & & & & & & - \\
\hline
\end{tabular}

Note*: M - arithmetic mean, SD - standard deviation, SEM - standard error of mean, Min - minimum value, Max - maximum value, P 0.025 percentile with 0.025 level, P 0.975 - percentile with 0.975 level.

\begin{tabular}{|c|c|c|c|c|c|c|c|c|}
\hline Element & $\mathbf{M}$ & SD & SEM & Min & Max & Median & P 0.025 & P 0.975 \\
\hline $\mathrm{Ag}$ & 0.263 & 0.285 & 0.09 & 0.002 & 0.842 & 0.157 & 0.014 & 0.811 \\
\hline $\mathrm{Al}$ & 17.9 & 6.7 & 3 & 9.8 & 26 & 16.4 & 10.2 & 25.7 \\
\hline As & $<0.004$ & - & - & - & - & - & - & - \\
\hline $\mathrm{Au}$ & 0.0097 & 0.0034 & 0.002 & 0.008 & 0.0148 & 0.008 & 0.008 & 0.0143 \\
\hline B & 1.8 & 0.45 & 0.2 & 1 & 2 & 2 & 1.1 & 2 \\
\hline $\mathrm{Be}$ & 0.0008 & 0.0004 & 0.0002 & 0.0002 & 0.001 & 0.001 & 0.00026 & 0.001 \\
\hline $\mathrm{Bi}$ & 0.049 & 0.042 & 0.021 & 0.0115 & 0.0959 & 0.0445 & 0.0119 & 0.0942 \\
\hline $\mathrm{Cd}$ & 1.44 & 0.99 & 0.44 & 0.26 & 3.01 & 1.31 & 0.362 & 2.84 \\
\hline $\mathrm{Ce}$ & 0.018 & 0.023 & 0.01 & 0.0031 & 0.0582 & 0.011 & 0.00334 & 0.0538 \\
\hline Co & 0.0667 & 0.0239 & 0.008 & 0.0387 & 0.123 & 0.0623 & 0.041 & 0.114 \\
\hline $\mathrm{Cr}$ & 1 & 1.17 & 0.39 & 0.234 & 3.65 & 0.617 & 0.244 & 3.36 \\
\hline Cs & 0.037 & 0.044 & 0.02 & 0.0095 & 0.114 & 0.0152 & 0.0099 & 0.106 \\
\hline Dy & $<0.005$ & - & - & - & - & - & - & - \\
\hline Er & 0.0048 & 0.0051 & 0.002 & 0.001 & 0.0138 & 0.003 & 0.0012 & 0.0127 \\
\hline $\mathrm{Eu}$ & $<0.001$ & - & - & - & - & - & - & - \\
\hline $\mathrm{Fe}$ & 434 & 744 & 224 & 96.5 & 2656 & 170 & 102 & 2088 \\
\hline Ga & $<0.019$ & - & - & - & - & - & - & - \\
\hline $\mathrm{Gd}$ & $<0.001$ & - & - & - & - & - & - & - \\
\hline $\mathrm{Hg}$ & 0.86 & 0.71 & 0.23 & 0.139 & 2.22 & 0.544 & 0.182 & 2.11 \\
\hline Ho & $<0.0002$ & - & - & - & - & - & - & - \\
\hline Ir & $<0.0003$ & - & - & - & - & - & - & - \\
\hline $\mathrm{La}$ & 0.0087 & 0.0086 & 0.004 & 0.0023 & 0.0237 & 0.0055 & 0.0025 & 0.0221 \\
\hline $\mathrm{Li}$ & 0.0273 & 0.0056 & 0.003 & 0.023 & 0.0355 & 0.0254 & 0.0231 & 0.0348 \\
\hline $\mathrm{Lu}$ & $<0.0002$ & - & - & - & - & - & - & - \\
\hline $\mathrm{Mn}$ & 2.32 & 1.73 & 0.71 & 0.45 & 5.5 & 1.86 & 0.578 & 5.16 \\
\hline Mo & 0.305 & 0.184 & 0.082 & 0.157 & 0.627 & 0.239 & 0.165 & 0.591 \\
\hline $\mathrm{Nb}$ & $<0.013$ & - & - & - & - & - & - & - \\
\hline $\mathrm{Nd}$ & 0.0183 & 0.0089 & 0.006 & 0.012 & 0.0246 & 0.0183 & 0.0123 & 0.0243 \\
\hline $\mathrm{Ni}$ & 4.08 & 3.86 & 1.7 & 0.9 & 10.4 & 3 & 0.94 & 9.84 \\
\hline $\mathrm{Pb}$ & 0.8 & 0.33 & 0.16 & 0.43 & 1.19 & 0.78 & 0.447 & 1.17 \\
\hline $\mathrm{Pd}$ & $<0.012$ & - & - & - & - & - & - & - \\
\hline
\end{tabular}




\begin{tabular}{|c|c|c|c|c|c|c|c|c|}
\hline $\operatorname{Pr}$ & 0.0054 & 0.0057 & 0.003 & 0.00053 & 0.0131 & 0.0041 & 0.00063 & 0.0126 \\
\hline $\mathrm{Pt}$ & $<0.0002$ & - & - & - & - & - & - & - \\
\hline $\mathrm{Rb}$ & 9.5 & 4.23 & 0.5 & 2.5 & 22.1 & 9.05 & 3.41 & 19.6 \\
\hline $\mathrm{Sb}$ & 0.124 & 0.085 & 0.027 & 0.03 & 0.339 & 0.114 & 0.035 & 0.296 \\
\hline $\mathrm{Sc}$ & 0.0088 & 0.0051 & 0.002 & 0.0016 & 0.0175 & 0.0071 & 0.0024 & 0.0172 \\
\hline Se & 3.94 & 3.72 & 1.18 & 1.22 & 13.8 & 2.92 & 1.28 & 12 \\
\hline Sm & 0.00142 & 0.0013 & 0.00058 & 0.0004 & 0.0037 & 0.001 & 0.00046 & 0.00343 \\
\hline Sn & 0.05 & 0.026 & 0.011 & 0.0243 & 0.0775 & 0.0504 & 0.0243 & 0.0771 \\
\hline $\mathrm{Tb}$ & $<0.0001$ & - & - & - & - & - & - & - \\
\hline $\mathrm{Te}$ & $<0.007$ & - & - & - & - & - & - & - \\
\hline Th & 0.006 & 0.0022 & 0.001 & 0.002 & 0.007 & 0.007 & 0.0025 & 0.007 \\
\hline $\mathrm{Ti}^{*}$ & $<0.4$ & - & - & - & - & - & - & - \\
\hline $\mathrm{Tl}$ & 0.00165 & 0.00065 & 0.00032 & 0.0011 & 0.0025 & 0.0015 & 0.00111 & 0.00245 \\
\hline $\mathrm{Tm}$ & $<0.0003$ & - & - & - & - & - & - & - \\
\hline $\mathrm{U}$ & $<0.0003$ & - & - & - & - & - & - & - \\
\hline Y & 0.00815 & 0.00007 & 0.00005 & 0.0081 & 0.0082 & 0.00815 & 0.0081 & 0.0082 \\
\hline $\mathrm{Yb}$ & $<0.0002$ & - & - & - & - & - & - & - \\
\hline $\mathrm{Zn}$ & 143 & 43 & 13 & 83 & 235 & 137 & 85.6 & 223 \\
\hline $\mathrm{Zr}$ & 0.086 & 0.031 & 0.014 & 0.031 & 0.1 & 0.1 & 0.038 & 0.1 \\
\hline
\end{tabular}

Note*: M - arithmetic mean, SD - standard deviation, SEM - standard error of mean, Min - minimum value, Max - maximum value, P 0.025 percentile with 0.025 level, P 0.975 - percentile with 0.975 level.

Table 5: Differences between mean values $(\mathrm{M} \pm \mathrm{SEM})$ of trace element mass fractions (mg/kg, dry mass basis) in normal thyroid and macro-follicular colloid nodular goiter.

\begin{tabular}{|c|c|c|c|c|c|}
\hline \multirow[b]{2}{*}{ Element } & \multicolumn{4}{|c|}{ Thyroid tissue } & \multirow{2}{*}{$\begin{array}{c}\text { Ratio } \\
\text { Goiter to Norm }\end{array}$} \\
\hline & $\begin{array}{l}\text { Normal thyroid } \\
n=105\end{array}$ & $\begin{array}{l}\text { Macro-follicular goiter } \\
\qquad n=16\end{array}$ & $\begin{array}{l}\text { Student's t-test } \\
\mathrm{p} \leq\end{array}$ & $\begin{array}{c}\text { U-test } \\
\text { p }\end{array}$ & \\
\hline $\mathrm{Ag}$ & $0.0133 \pm 0.0013$ & $0.108 \pm 0.035$ & 0.022 & $\leq 0.01$ & 8.12 \\
\hline $\mathrm{Al}$ & $10.5 \pm 1.8$ & $26.6 \pm 7.2$ & 0.05 & $\leq 0.01$ & 2.53 \\
\hline $\mathrm{B}$ & $0.476 \pm 0.058$ & $1.24 \pm 0.13$ & 0.00008 & $\leq 0.01$ & 2.61 \\
\hline $\mathrm{Be}$ & $0.00052 \pm 0.00008$ & $0.00055 \pm 0.00009$ & 0.799 & $>0.05$ & 1.06 \\
\hline $\mathrm{Bi}$ & $0.0072 \pm 0.0022$ & $0.056 \pm 0.019$ & 0.027 & $\leq 0.01$ & 7.78 \\
\hline $\mathrm{Cd}$ & $2.08 \pm 0.27$ & $1.07 \pm 0.40$ & 0.05 & $\leq 0.01$ & 0.51 \\
\hline $\mathrm{Ce}$ & $0.0080 \pm 0.0011$ & $0.0189 \pm 0.0050$ & 0.073 & $>0.05$ & 2.36 \\
\hline Co & $0.0390 \pm 0.0031$ & $0.0495 \pm 0.0068$ & 0.176 & $>0.05$ & 1.27 \\
\hline $\mathrm{Cr}$ & $0.495 \pm 0.031$ & $1.39 \pm 0.45$ & 0.065 & $>0.05$ & 2.81 \\
\hline $\mathrm{Cs}$ & $0.0245 \pm 0.0022$ & $0.0159 \pm 0.0020$ & 0.0051 & $\leq 0.01$ & 0.65 \\
\hline Er & $0.000377 \pm 0.000050$ & $0.00167 \pm 0.00026$ & 0.00035 & $\leq 0.01$ & 4.43 \\
\hline $\mathrm{Fe}$ & $222.8 \pm 9.6$ & $431 \pm 98$ & 0.05 & $\leq 0.01$ & 1.93 \\
\hline $\mathrm{Ga}$ & $0.0316 \pm 0.0021$ & $0.0203 \pm 0.0030$ & 0.0078 & $\leq 0.01$ & 0.64 \\
\hline $\mathrm{Hg}$ & $0.0543 \pm 0.0043$ & $1.43 \pm 0.35$ & 0.0014 & $\leq 0.01$ & 26.3 \\
\hline $\mathrm{La}$ & $0.00475 \pm 0.00062$ & $0.0097 \pm 0.0030$ & 0.107 & $>0.05$ & 2.04 \\
\hline $\mathrm{Li}$ & $0.0208 \pm 0.0022$ & $0.0268 \pm 0.0040$ & 0.221 & $>0.05$ & 1.29 \\
\hline $\mathrm{Mn}$ & $1.28 \pm 0.07$ & $1.35 \pm 0.18$ & 0.73 & $>0.05$ & 1.05 \\
\hline Mo & $0.0836 \pm 0.0062$ & $0.165 \pm 0.019$ & 0.0012 & $\leq 0.01$ & 1.97 \\
\hline $\mathrm{Nd}$ & $0.0041 \pm 0.0004$ & $0.0131 \pm 0.0030$ & 0.009 & $\leq 0.01$ & 3.2 \\
\hline $\mathrm{Ni}$ & $0.449 \pm 0.046$ & $2.55 \pm 0.46$ & 0.00082 & $\leq 0.01$ & 5.68 \\
\hline $\mathrm{Pb}$ & $0.233 \pm 0.033$ & $0.383 \pm 0.058$ & 0.037 & $\leq 0.01$ & 1.64 \\
\hline
\end{tabular}




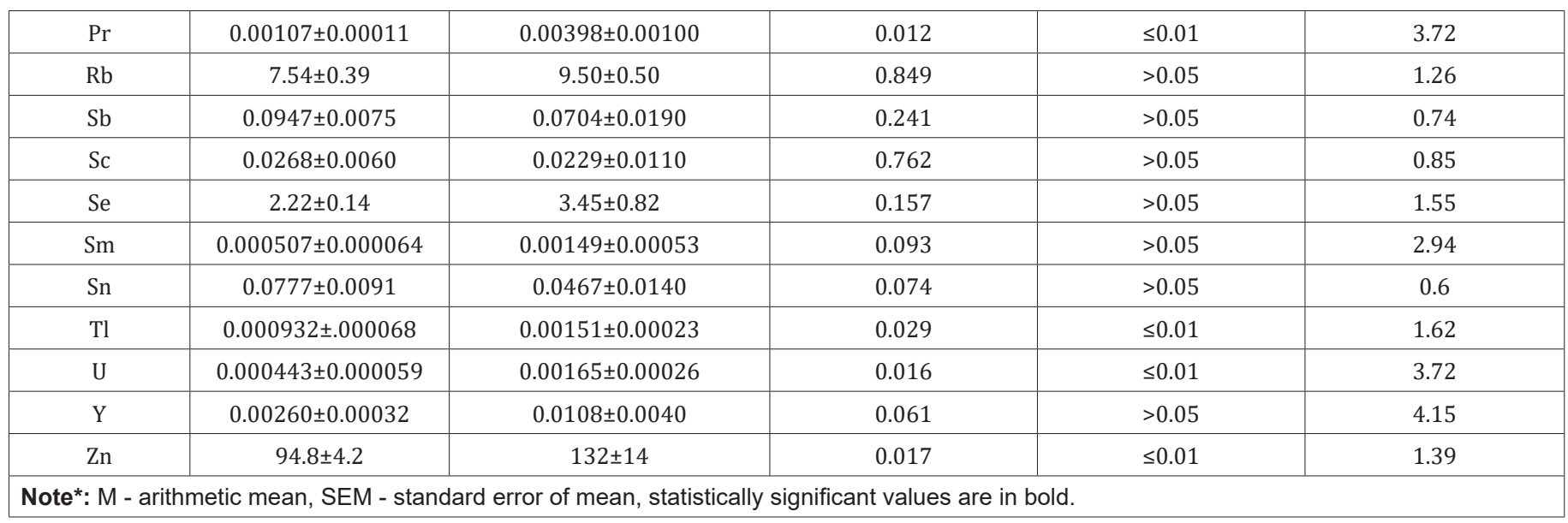

Table 6: Differences between mean values $(\mathrm{M} \pm \mathrm{SEM})$ of trace element mass fractions ( $\mathrm{mg} / \mathrm{kg}$, dry mass basis) in normal thyroid and micro-follicular colloid nodular goiter.

\begin{tabular}{|c|c|c|c|c|c|}
\hline \multirow{2}{*}{ Element } & \multicolumn{4}{|c|}{ Thyroid tissue } & \multirow{2}{*}{$\begin{array}{c}\text { Ratio } \\
\text { Goiter to Norm }\end{array}$} \\
\hline & $\begin{array}{l}\text { Normal thyroid } \\
n=105\end{array}$ & $\begin{array}{l}\text { Micro-follicular goiter } \\
n=13\end{array}$ & $\begin{array}{c}\text { Student's t-test } \\
\mathrm{p} \leq\end{array}$ & $\begin{array}{c}\text { U-test } \\
\mathbf{p}\end{array}$ & \\
\hline $\mathrm{Ag}$ & $0.0133 \pm 0.0013$ & $0.263 \pm 0.090$ & 0.021 & $\leq 0.01$ & 19.8 \\
\hline $\mathrm{Al}$ & $10.5 \pm 1.8$ & $17.9 \pm 3.0$ & 0.066 & $>0.05$ & 1.7 \\
\hline $\mathrm{B}$ & $0.476 \pm 0.058$ & $1.80 \pm 0.20$ & 0.0018 & $\leq 0.01$ & 3.78 \\
\hline $\mathrm{Be}$ & $0.00052 \pm 0.00008$ & $0.00080 \pm 0.00020$ & 0.257 & $>0.05$ & 1.54 \\
\hline $\mathrm{Bi}$ & $0.0072 \pm 0.0022$ & $0.049 \pm 0.021$ & 0.138 & $>0.05$ & 6.81 \\
\hline $\mathrm{Cd}$ & $2.08 \pm 0.27$ & $1.44 \pm 0.44$ & 0.257 & $>0.05$ & 0.69 \\
\hline $\mathrm{Ce}$ & $0.0080 \pm 0.0011$ & $0.0180 \pm 0.0100$ & 0.362 & $>0.05$ & 2.25 \\
\hline Co & $0.0390 \pm 0.0031$ & $0.0667 \pm 0.0080$ & 0.0083 & $\leq 0.01$ & 1.71 \\
\hline $\mathrm{Cr}$ & $0.495 \pm 0.031$ & $1.00 \pm 0.39$ & 0.232 & $>0.05$ & 2.02 \\
\hline Cs & $0.0245 \pm 0.0022$ & $0.0370 \pm 0.0200$ & 0.553 & $>0.05$ & 1.51 \\
\hline Er & $0.000377 \pm 0.000050$ & $0.00480 \pm 0.00200$ & 0.129 & $>0.05$ & 12.7 \\
\hline $\mathrm{Fe}$ & $222.8 \pm 9.6$ & $434 \pm 224$ & 0.369 & $>0.05$ & 1.95 \\
\hline Ga & $0.0316 \pm 0.0021$ & $<0.019$ & - & - & - \\
\hline $\mathrm{Hg}$ & $0.0543 \pm 0.0043$ & $0.86 \pm 0.23$ & $<0.0061$ & $\leq 0.01$ & 15.8 \\
\hline $\mathrm{La}$ & $0.00475 \pm 0.00062$ & $0.00870 \pm 0.00400$ & 0.368 & $>0.05$ & 1.83 \\
\hline $\mathrm{Li}$ & $0.0208 \pm 0.0022$ & $0.0273 \pm 0.0030$ & 0.107 & $>0.05$ & 1.31 \\
\hline $\mathrm{Mn}$ & $1.28 \pm 0.07$ & $2.32 \pm 0.71$ & 0.202 & $>0.05$ & 1.81 \\
\hline Mo & $0.0836 \pm 0.0062$ & $0.305 \pm 0.082$ & 0.054 & $\leq 0.05$ & 3.65 \\
\hline $\mathrm{Nd}$ & $0.0041 \pm 0.0004$ & $0.0183 \pm 0.0060$ & 0.264 & $>0.05$ & 4.46 \\
\hline $\mathrm{Ni}$ & $0.449 \pm 0.046$ & $4.08 \pm 1.7$ & 0.103 & $>0.05$ & 9.09 \\
\hline $\mathrm{Pb}$ & $0.233 \pm 0.033$ & $0.800 \pm 0.160$ & 0.038 & $\leq 0.01$ & 3.43 \\
\hline $\operatorname{Pr}$ & $0.00107 \pm 0.00011$ & $0.00540 \pm 0.00300$ & 0.22 & $>0.05$ & 5.05 \\
\hline $\mathrm{Rb}$ & $7.54 \pm 0.39$ & $9.50 \pm 0.50$ & 0.027 & $\leq 0.01$ & 1.26 \\
\hline $\mathrm{Sb}$ & $0.0947 \pm 0.0075$ & $0.124 \pm 0.027$ & 0.322 & $>0.05$ & 1.31 \\
\hline Sc & $0.0268 \pm 0.0060$ & $0.0088 \pm 0.0020$ & 0.0067 & $\leq 0.01$ & 0.33 \\
\hline $\mathrm{Se}$ & $2.22 \pm 0.14$ & $3.94 \pm 1.18$ & 0.179 & $>0.05$ & 1.77 \\
\hline $\mathrm{Sm}$ & $0.000507 \pm 0.000064$ & $0.00142 \pm 0.00058$ & 0.192 & $>0.05$ & 2.8 \\
\hline Sn & $0.0777 \pm 0.0091$ & $0.0500 \pm 0.0110$ & 0.088 & $>0.05$ & 0.64 \\
\hline $\mathrm{Tl}$ & $0.000932 \pm .000068$ & $0.00165 \pm 0.00032$ & 0.11 & $>0.05$ & 1.77 \\
\hline $\mathrm{U}$ & $0.000443 \pm 0.000059$ & $<0.0003$ & - & - & - \\
\hline
\end{tabular}




\begin{tabular}{|c|c|c|c|c|c|}
\hline Y & $0.00260 \pm 0.00032$ & $0.00815 \pm 0.00005$ & 0.0001 & $\leq 0.01$ & 3.13 \\
\hline $\mathrm{Zn}$ & $94.8 \pm 4.2$ & $143 \pm 13$ & 0.0042 & $\leq 0.01$ & 1.51 \\
\hline
\end{tabular}

\begin{tabular}{|c|c|c|c|c|c|}
\hline \multirow[t]{2}{*}{ Element } & \multicolumn{4}{|c|}{ Thyroid tissue } & \multirow{2}{*}{$\begin{array}{c}\text { Ratio } \\
\begin{array}{c}\text { Macro- to Micro- } \\
\text { follicular }\end{array} \\
\end{array}$} \\
\hline & $\begin{array}{l}\text { Macro-follicular goiter } \\
n=16\end{array}$ & $\begin{array}{l}\text { Micro-follicular goiter } \\
n=13\end{array}$ & $\begin{array}{l}\text { Student's t-test } \\
\mathrm{p} \leq\end{array}$ & $\begin{array}{c}\text { U-test } \\
\mathbf{p}\end{array}$ & \\
\hline $\mathrm{Ag}$ & $0.108 \pm 0.035$ & $0.263 \pm 0.090$ & 0.134 & $>0.05$ & 0.41 \\
\hline $\mathrm{Al}$ & $26.6 \pm 7.2$ & $17.9 \pm 3.0$ & 0.286 & $>0.05$ & 1.49 \\
\hline B & $1.24 \pm 0.13$ & $1.80 \pm 0.20$ & 0.049 & $\leq 0.01$ & 0.69 \\
\hline $\mathrm{Be}$ & $0.00055 \pm 0.00009$ & $0.00080 \pm 0.00020$ & 0.309 & $>0.05$ & 0.69 \\
\hline $\mathrm{Bi}$ & $0.056 \pm 0.019$ & $0.049 \pm 0.021$ & 0.822 & $>0.05$ & 1.14 \\
\hline $\mathrm{Cd}$ & $1.07 \pm 0.40$ & $1.44 \pm 0.44$ & 0.548 & $>0.05$ & 0.74 \\
\hline $\mathrm{Ce}$ & $0.0189 \pm 0.0050$ & $0.018 \pm 0.010$ & 0.973 & $>0.05$ & 1.05 \\
\hline Co & $0.0495 \pm 0.0068$ & $0.0667 \pm 0.0080$ & 0.117 & $>0.05$ & 0.74 \\
\hline $\mathrm{Cr}$ & $1.39 \pm 0.45$ & $1.00 \pm 0.39$ & 0.514 & $>0.05$ & 1.39 \\
\hline Cs & $0.0159 \pm 0.0020$ & $0.037 \pm 0.020$ & 0.337 & $>0.05$ & 0.43 \\
\hline $\mathrm{Er}$ & $0.00167 \pm 0.00026$ & $0.0048 \pm 0.0020$ & 0.249 & $>0.05$ & 0.35 \\
\hline $\mathrm{Fe}$ & $431 \pm 98$ & $434 \pm 224$ & 0.99 & $>0.05$ & 0.99 \\
\hline Ga & $0.0203 \pm 0.0030$ & $<0.019$ & - & - & - \\
\hline $\mathrm{Hg}$ & $1.43 \pm 0.35$ & $0.86 \pm 0.23$ & 0.178 & $>0.05$ & 1.66 \\
\hline La & $0.0097 \pm 0.0030$ & $0.0087 \pm 0.0040$ & 0.841 & $>0.05$ & 1.11 \\
\hline $\mathrm{Li}$ & $0.0268 \pm 0.0040$ & $0.0273 \pm 0.0030$ & 0.919 & $>0.05$ & 0.98 \\
\hline $\mathrm{Mn}$ & $1.35 \pm 0.18$ & $2.32 \pm 0.71$ & 0.233 & $>0.05$ & 0.58 \\
\hline Mo & $0.165 \pm 0.019$ & $0.305 \pm 0.082$ & 0.165 & $>0.05$ & 0.54 \\
\hline $\mathrm{Nd}$ & $0.0131 \pm 0.0030$ & $0.0183 \pm 0.0060$ & 0.554 & $>0.05$ & 0.72 \\
\hline $\mathrm{Ni}$ & $2.55 \pm 0.46$ & $4.08 \pm 1.7$ & 0.433 & $>0.05$ & 0.63 \\
\hline $\mathrm{Pb}$ & $0.383 \pm 0.058$ & $0.80 \pm 0.16$ & 0.079 & $\leq 0.05$ & 0.48 \\
\hline $\operatorname{Pr}$ & $0.00398 \pm 0.00100$ & $0.0054 \pm 0.0030$ & 0.654 & $>0.05$ & 0.74 \\
\hline $\mathrm{Rb}$ & $9.50 \pm 0.50$ & $9.50 \pm 0.50$ & 0.954 & $>0.05$ & 1 \\
\hline $\mathrm{Sb}$ & $0.0704 \pm 0.0190$ & $0.124 \pm 0.027$ & 0.121 & $>0.05$ & 0.57 \\
\hline $\mathrm{Sc}$ & $0.0229 \pm 0.0110$ & $0.0088 \pm 0.0020$ & 0.239 & $>0.05$ & 2.6 \\
\hline $\mathrm{Se}$ & $3.45 \pm 0.82$ & $3.94 \pm 1.18$ & 0.739 & $>0.05$ & 0.88 \\
\hline $\mathrm{Sm}$ & $0.00149 \pm 0.00053$ & $0.00142 \pm 0.00058$ & 0.936 & $>0.05$ & 1.05 \\
\hline Sn & $0.0467 \pm 0.0140$ & $0.050 \pm 0.011$ & 0.852 & $>0.05$ & 0.93 \\
\hline $\mathrm{Tl}$ & $0.00151 \pm 0.00023$ & $0.00165 \pm 0.00032$ & 0.742 & $>0.05$ & 0.92 \\
\hline $\mathrm{U}$ & $0.00165 \pm 0.00026$ & $<0.0003$ & - & - & - \\
\hline Y & $0.0108 \pm 0.0040$ & $0.00815 \pm 0.00005$ & 0.497 & $>0.05$ & 1.33 \\
\hline $\mathrm{Zn}$ & $132 \pm 14$ & $143 \pm 13$ & 0.591 & $>0.05$ & 0.92 \\
\hline
\end{tabular}

\section{Discussion}

\section{Precision And Accuracy of Results}

A good agreement of our results for the TE mass fractions with the certified values of CRM IAEA H-4, CRM IAEA HH-1, INCTSBF-4, INCT-TL-1, and INCT-MPH-2 [29,30,35,50-55] as well as the similarity of the means of the $\mathrm{Ag}$, $\mathrm{Co}, \mathrm{Cr}, \mathrm{Fe}, \mathrm{Hg}, \mathrm{Rb}, \mathrm{Sb}, \mathrm{Se}$, and $\mathrm{Zn}$ mass fractions in the normal human thyroid determined by both INAA-LLR and ICP-MS methods (Table 1) demonstrates an acceptable precision and accuracy of the results obtained in the study and presented in Tables 2-7. 


\section{Effect of Goitrous Transformation on TE Contents}

From Table 5, it is observed that in macro-follicular CNG the mass fraction of $\mathrm{Ag}, \mathrm{Al}, \mathrm{B}, \mathrm{Bi}, \mathrm{Er}, \mathrm{Fe}, \mathrm{Hg}, \mathrm{Mo}, \mathrm{Nd}, \mathrm{Ni}, \mathrm{Pb}, \mathrm{Pr}, \mathrm{Tl}, \mathrm{U}$, and $\mathrm{Zn}$ is $8.12,2.53,2.61,7.78,4.43,1.93,26.3,1.97,3.20,5.68,1.64$ $3.72,1.62,3.72$ and 1.39 times, respectively, higher, whereas the mass fraction of $\mathrm{Cd}, \mathrm{Cs}$, and $\mathrm{Ga}$ is $49 \%, 35 \%$, and $36 \%$, respectively, lower than in tissues of the normal thyroid. From Table 6 , it is observed that in micro-follicular CNG the mass fraction of Ag, B, Co, $\mathrm{Hg}$, Mo, $\mathrm{Pb}, \mathrm{Rb}, \mathrm{Y}$, and $\mathrm{Zn}$ is $19.8,3.78,1.71,15.9,3.65,3.43,1.26$, 3.13 , and 1.51 times, respectively, higher, whereas the mass fraction of Sc is $67 \%$ lower than in tissues of the normal thyroid. Thus, if we accept the TE contents in thyroid glands in the control group as a norm, we must conclude that with a goitrous transformation the $\mathrm{Ag}, \mathrm{Al}, \mathrm{B}, \mathrm{Bi}, \mathrm{Co}, \mathrm{Er}, \mathrm{Fe}, \mathrm{Hg}, \mathrm{Mo}, \mathrm{Nd}, \mathrm{Ni}, \mathrm{Pb}, \mathrm{Pr}, \mathrm{Rb}, \mathrm{Tl}, \mathrm{U}, \mathrm{Y}$, and Zn level in thyroid tissue can be significantly changed.

\section{Association Between TE Levels and Relative Volume of Colloid and Cells}

Comparison mass fraction of $\mathrm{Ag}, \mathrm{Al}, \mathrm{B}, \mathrm{Be}, \mathrm{Bi}, \mathrm{Cd}, \mathrm{Ce}, \mathrm{Co}, \mathrm{Cr}, \mathrm{Cs}$, Er, Fe, Ga, Hg, La, Li, Mn, Mo, Nd, Ni, Pb, Pr, Rb, Sb, Sc, Se, Sm, Sn, Tl, $\mathrm{U}, \mathrm{Y}$, and $\mathrm{Zn}$ in macro- and micro-follicular CNG shown that level of $\mathrm{B}$ and $\mathrm{Pb}$ in macro-follicular goiter is $31 \%$ and $52 \%$, respectively, lower than in micro-follicular goiter (Table 7). Because the relative volume of cells in the micro-follicular CNG is higher than in the macro-follicular $\mathrm{CNG}$, it is possible to conclude that $\mathrm{B}$ and $\mathrm{Pb}$ increasingly associated with thyroid cells.

\section{Comparison With Published Data}

The published data on TE contents in the CNG in comparison with normal levels are very scanty and contradictory. For example, information on B, Bi, Ce, Cs, Er, Ga, La, Li, Nd, Pr, Sb, Sc, Sm, Sn, Tl, and Y content in CNG was not found. Kovalev [58] found elevated levels of Ag in the CNG, but Gudzhedzhiani [59] did not. In study of Kamenev [60] the Al content in goitrous tissue was twice as much as the normal level, but Antonova, et al. [61] did not find any difference in $\mathrm{Al}$ content, whereas Ianchur, et al. [62], Elenevskaia, et al. [63], Bredikhin and Soroka [64], and Li [65] demonstrated a decrease of this metal mass fraction in goiter. A significant decrease of the $\mathrm{Zn}$ content during goitrous transformation was shown by Błazewicz et al. [66], but in the recent study this change was not confirmed [9]. Information on the TE contents in macro- or microfollicular CNG, as well as about the association between TE level and relative volume of colloid and cells in goitrous tissue was not found.

\section{Limitations}

This study has several limitations. Firstly, analytical techniques employed in this study measure only fifty TE mass fractions. Future studies should be directed toward using other analytical methods which will extend the list of TE investigated in normal and goitrous thyroid. Secondly, the sample size of macro- or micro-follicular CNG groups was relatively small and prevented investigations of TE contents in CNG group using differentials like gender, stage of disease, and dietary habits of healthy persons and patients with CNG. Lastly, generalization of our results may be limited to Russian population. Despite these limitations, this study provides evidence on goiter-specific tissue of $\mathrm{Ag}, \mathrm{Al}, \mathrm{B}, \mathrm{Bi}, \mathrm{Co}, \mathrm{Er}, \mathrm{Fe}, \mathrm{Hg}$, Mo, $\mathrm{Nd}, \mathrm{Ni}, \mathrm{Pb}$, $\mathrm{Pr}, \mathrm{Rb}, \mathrm{Tl}, \mathrm{U}, \mathrm{Y}$, and $\mathrm{Zn}$ level alteration, demonstrates associations between $\mathrm{B}$ and $\mathrm{Pb}$ content and relative volume of cells in $\mathrm{CNG}$, and shows the necessity to continue TE research of CNG of different histology.

\section{Conclusion}

In this work, TE analysis was carried out in the tissue samples of normal and goitrous thyroid using a combination of nondestructive INAA-LLR and destructive ICP-MS. It was shown that this combination is an adequate analytical tool for the estimation of fifty TE (Ag, Al, As, Au, B, Be, Bi, Cd, Ce, Co, Cr, Cs, Dy, Er, Eu, Ga, Gd, Hg, Ho, Ir, La, Li, Lu, Mn, Mo, Nb, Nd, Ni, Pb, Pd, Pr, Pt, Rb, Sb, $\mathrm{Se}, \mathrm{Sm}, \mathrm{Sn}, \mathrm{Tb}, \mathrm{Te}, \mathrm{Th}, \mathrm{Ti}, \mathrm{Tl}, \mathrm{Tm}, \mathrm{U}, \mathrm{Y}, \mathrm{Yb}, \mathrm{Zn}$, and $\mathrm{Zr}$ ) content in the tissue samples of human thyroid in norm and pathology, including needle-biopsy cores. It was observed the considerable changes in TE contents in the goitrous transformed tissue of thyroid, which depend on the histology of goiter. It was found that $\mathrm{B}$ and $\mathrm{Pb}$ predominately accumulates in cells of CNG.

\section{Acknowledgements}

The author is extremely grateful to Profs. Vtyurin BM and MedvedevVS, Medical Radiological Research Center, Obninsk, as well as to Dr. Choporov Yu, Head of the Forensic Medicine Department of City Hospital, Obninsk, for supplying thyroid samples. The author is also grateful to Dr. Karandaschev V, Dr. Nosenko S, and Moskvina I, Institute of Microelectronics Technology and High Purity Materials, Chernogolovka, Russia, for their help in ICP-MS analysis.

\section{Conflicts of Interest}

The authors declare that they have no financial conflicts of interest.

\section{Funding}

There were not any sources of funding that have supported this work.

\section{References}

1. Stuchi LP, Castanhole Nunes MMU, Maniezzo Stuchi N, Patrícia M Biselli Chicote, Tiago Henrique, et al. (2020) EGFA and NFE2L2 gene expression and regulation by microRNAs in thyroid papillary cancer and colloid goiter. Genes (Basel) 11(9): 954.

2. Campbell MJ, Seib CD, Candell L, Jessica E Gosnell, Quan Yang Duh, et al. (2015) The underestimated risk of cancer in patients with multinodular goiters after a benign fine needle aspiration. World J Surg 39(3): 695700 . 
3. Frilling A, Liu C, Weber F (2004) Benign multinodular goiter. Scan J Surg 93(4): 278-281.

4. Derwahl M, Studer H (2000) Multinodular goitre: 'much more to it than simply iodine deficiency'. Baillieres Best Pract Res Clin Endocrinol Metab 14(4): 577-600.

5. Zaichick V (1998) Iodine excess and thyroid cancer. J Trace Elem Exp Med 11(4): 508-509.

6. Zaichick V, Iljina T (1998) Dietary iodine supplementation effect on the rat thyroid 131I blastomogenic action. In: Die Bedentung der Mengenund Spurenelemente. 18. Arbeitstangung. Friedrich-Schiller-Universität pp. 294-306.

7. Kim S, Kwon YS, Kim JY, Kyung Hee Hong, Yoo Kyoung Park (2019) Association between Iodine Nutrition Status and Thyroid DiseaseRelated Hormone in Korean Adults: Korean National Health and Nutrition Examination Survey VI (2013-2015). Nutrients 11(11): 2757.

8. Vargas Uricoechea P, Pinzón Fernández MV, Bastidas Sánchez BE, Elisa Jojoa Tobar, Luis Eduardo Ramírez Bejarano, et al. (2019) Iodine status in the colombian population and the impact of universal salt iodization: a double-edged sword? J Nutr Metab 2019: 6239243.

9. Stojsavljević A, Rovčanin B, Krstić D, Slavica Borković Mitić, Ivan Paunović, et al. (2019) Risk assessment of toxic and essential trace metals on the thyroid health at the tissue level: The significance of lead and selenium for colloid goiter disease. Expo Health 12: 255-264.

10. Fahim YA, Sharaf NE, Hasani IW, Eman A Ragab, Heba K Abdelhakim (2020) Assessment of thyroid function and oxidative stress state in foundry workers exposed to lead. J Health Pollut 10(27): 200903.

11. Liu M, Song J, Jiang Y, Yuan Liu, Jinling Peng, et al. (2021) A case-control study on the association of mineral elements exposure and thyroid tumor and goiter. Ecotoxicol Environ Saf 208: 111615.

12. Zaichick V (2006) Medical elementology as a new scientific discipline. J Radioanal Nucl Chem 269: 303-309.

13. Moncayo R, Moncayo H (2017) A post-publication analysis of the idealized upper reference value of $2.5 \mathrm{mIU} / \mathrm{L}$ for TSH: Time to support the thyroid axis with magnesium and iron especially in the setting of reproduction medicine. BBA Clin 197: 115-119.

14. Beyersmann D, Hartwig A (2008) Carcinogenic metal compounds: recent insight into molecular and cellular mechanisms. Arch Toxicol 82(8): 493-512.

15. Martinez Zamudio R, Ha HC (2011) Environmental epigenetics in metal exposure. Epigenetics 6(7): 820-827.

16. Zaĭchik VE, Raibukhin YuS, Melnik AD, V I Cherkashin (1970) Neutronactivation analysis in the study of the behavior of iodine in the organism. Med Radiol (Mosk) 15(1): 33-36.

17. Zaĭchik VE, Matveenko EG, Vtiurin BM, V S Medvedev (1982) Intrathyroid iodine in the diagnosis of thyroid cancer. Vopr Onkol 28(3): 18-24.

18. Zaichick V, Tsyb AF, Vtyurin BM (1995) Trace elements and thyroid cancer. Analyst 120(3): 817-821.

19. Zaichick VYe, Choporov YuYa (1996) Determination of the natural level of human intra-thyroid iodine by instrumental neutron activation analysis. J Radioanal Nucl Chem 207(1): 153-161.

20. Zaichick V (1998) In vivo and in vitro application of energy dispersive $\mathrm{XRF}$ in clinical investigations: experience and the future. J Trace Elem Exp Med 11(4): 509-510.

21. Zaichick V, Zaichick S (1999) Energy-dispersive X-ray fluorescence of iodine in thyroid puncture biopsy specimens. J Trace Microprobe Tech 17(2): 219-232.

22. Zaichick V (2000) Relevance of, and potentiality for in vivo intrathyroidal iodine determination. Ann N Y Acad Sci 904: 630-632.
23. Zaichick V, Zaichick S (1997) Normal human intrathyroidal iodine. Sci Total Environ 206(1): 39-56.

24. Zaichick V (1999) Human intrathyroidal iodine in health and nonthyroidal disease. In: New aspects of trace element research (Eds: M.Abdulla, M.Bost, S.Gamon, P.Arnaud, G.Chazot). Smith-Gordon, London, and Nishimura,Tokyo, pp. 114-119.

25. Zaichick V, Zaichick S (2017) Age-related changes of some trace element contents in intact thyroid of females investigated by energy dispersive X-ray fluorescent analysis. Trends Geriatr Healthc 1(1): 31-38.

26. Zaichick V, Zaichick S (2017) Age-related changes of some trace element contents in intact thyroid of males investigated by energy dispersive X-ray fluorescent analysis. MOJ Gerontol Ger 1(5): 133-140.

27. Zaichick V, Zaichick S (2017) Age-related changes of $\mathrm{Br}, \mathrm{Ca}, \mathrm{Cl}, \mathrm{I}, \mathrm{K}, \mathrm{Mg}$ $\mathrm{Mn}$, and Na contents in intact thyroid of females investigated by neutron activation analysis. Curr Updates Aging 1: 5.1.

28. Zaichick V, Zaichick S (2017) Age-related changes of $\mathrm{Br}, \mathrm{Ca}, \mathrm{Cl}, \mathrm{I}, \mathrm{K}, \mathrm{Mg}$, $\mathrm{Mn}$, and $\mathrm{Na}$ contents in intact thyroid of males investigated by neutron activation analysis. J Aging Age Relat Dis 1(1): 1002.

29. Zaichick V, Zaichick S (2017) Age-related changes of Ag, Co, Cr, Fe, Hg $\mathrm{Rb}, \mathrm{Sb}, \mathrm{Sc}, \mathrm{Se}$, and $\mathrm{Zn}$ contents in intact thyroid of females investigated by neutron activation analysis. J Gerontol Geriatr Med 3: 015.

30. Zaichick V, Zaichick S (2017) Age-related changes of Ag, Co, Cr, Fe, Hg $\mathrm{Rb}, \mathrm{Sb}, \mathrm{Sc}, \mathrm{Se}$, and $\mathrm{Zn}$ contents in intact thyroid of males investigated by neutron activation analysis. Curr Trends Biomedical Eng Biosci 4(4): 64-67.

31. Zaichick V, Zaichick S (2018) Effect of age on chemical element contents in female thyroid investigated by some nuclear analytical methods. MicroMedicine 6(1): 47-61

32. Zaichick V, Zaichick S (2018) Neutron activation and X-ray fluorescent analysis in study of association between age and chemical element contents in thyroid of males. Op Acc J Bio Eng Bio Sci 2(4): 202-212.

33. Zaichick V, Zaichick S (2018) Variation with age of chemical element contents in females' thyroids investigated by neutron activation analysis and inductively coupled plasma atomic emission spectrometry. J Biochem Analyt Stud 3(1): 1-10.

34. Zaichick V, Zaichick S 92018) Association between age and twenty chemical element contents in intact thyroid of males. SM Gerontol Geriatr Res 2(1): 1014

35. Zaichick V, Zaichick S (2018) Associations between age and 50 trace element contents and relationships in intact thyroid of males. Aging Clin Exp Res 30(9): 1059-1070.

36. Zaichick V, Zaichick S (2018) Possible role of inadequate quantities of intra-thyroidal bromine, rubidium and zinc in the etiology of female subclinical hypothyroidism. EC Gynaecology 7(3): 107-115.

37. Zaichick V, Zaichick S (2018) Possible role of inadequate quantities of intra-thyroidal bromine, calcium and magnesium in the etiology of female subclinical hypothyroidism. Int Gyn and Women's Health 1(3): IGWHC.MS.ID.000113.

38. Zaichick V, Zaichick S (2018) Possible role of inadequate quantities of intra-thyroidal cobalt, rubidium and zinc in the etiology of female subclinical hypothyroidism. Womens Health Sci J 2(1): 000108.

39. Zaichick V, Zaichick S (2018) Association between female subclinical hypothyroidism and inadequate quantities of some intra-thyroidal chemical elements investigated by X-ray fluorescence and neutron activation analysis. Gynaecology and Perinatology 2(4): 340-355.

40. Zaichick V, Zaichick S (2018) Investigation of association between the high risk of female subclinical hypothyroidism and inadequate quantities of twenty intra-thyroidal chemical elements. Clin Res: Gynecol Obstet 1(1): 1-18. 
41. Zaichick V, Zaichick S (2018) Investigation of association between the high risk of female subclinical hypothyroidism and inadequate quantities of intra-thyroidal trace elements using neutron activation and inductively coupled plasma mass spectrometry. Acta Scientific Medical Sciences 2(9): 23-37.

42. Zaichick V, Zaichick S (2018) Trace element contents in thyroid cancer investigated by energy dispersive X-ray fluorescent analysis. American Journal of Cancer Research and Reviews 2: 5.

43. Zaichick V, Zaichick S (2018) Trace element contents in thyroid cancer investigated by instrumental neutron activation analysis. J Oncol Res 2(1): 1-13.

44. Zaichick V, Zaichick S (2018) Variation in selected chemical element contents associated with malignant tumors of human thyroid gland Cancer Studies 2(1): 2

45. Zaichick V, Zaichick S (2018) Twenty chemical element contents in normal and cancerous thyroid. Int J Hematol Blo Dis 3(2): 1-13.

46. Zaichick V, Zaichick S (2018) Levels of chemical element contents in thyroid as potential biomarkers for cancer diagnosis (a preliminary study). J Cancer Metastasis Treat 4: 60 .

47. Zaichick V, Zaichick S (2018) Fifty trace element contents in normal and cancerous thyroid. Acta Scientific Cancer Biology 2(8): 21-38.

48. Zaichick V, Zaichick S (1996) Instrumental effect on the contamination of biomedical samples in the course of sampling. The Journal of Analytical Chemistry 51(12): 1200-1205

49. Zaichick V, Zaichick S (1997) A search for losses of chemical elements during freeze-drying of biological materials. J Radioanal Nucl Chem 218(2): 249-253.

50. Zaichick S, Zaichick V (2011) The effect of age on $\mathrm{Ag}, \mathrm{Co}, \mathrm{Cr}, \mathrm{Fe}, \mathrm{Hg}, \mathrm{Sb}$, $\mathrm{Sc}, \mathrm{Se}$, and $\mathrm{Zn}$ contents in intact human prostate investigated by neutron activation analysis. Appl Radiat Isot 69(6): 827-833.

51. Zaichick S, Zaichick V (2010) The effect of age and gender on 37 chemical element contents in scalp hair of healthy humans. Biol Trace Elem Res 134(1): 41-54.

52. Zaichick S, Zaichick V, Nosenko S, Irina Moskvina (2012) Mass fractions of 52 trace elements and zinc trace element content ratios in intact human prostates investigated by inductively coupled plasma mass spectrometry. Biol Trace Elem Res 149(2): 171-183.

53. Zaichick V, Zaichick S (2013) Use of neutron activation analysis and inductively coupled plasma mass spectrometry for the determination of trace elements in pediatric and young adult prostate. AJAC 4: 696-706.
54. Zaichick V, Zaichick S (2014) Use of INAA and ICP-MS for the assessment of trace element mass fractions in adult and geriatric prostate. J Radioanal Nucl Chem 301(2): 383-397

55. Zaichick S, Zaichick V (2010) The effect of age and gender on 37 chemical element contents in scalp hair of healthy humans. Biol Trace Elem Res 134(1): 41-54.

56. Zaichick V (1995) Applications of synthetic reference materials in the medical Radiological Research Centre. Fresenius J Anal Chem 352: 219223.

57. Korelo AM, Zaichick V (1993) Software to optimize the multielement INAA of medical and environmental samples. In: Activation Analysis in Environment Protection. Joint Institute for Nuclear Research, Dubna pp. 326-332.

58. Kovalev MM (1960) Trace element contents in normal and goitrous glands. Vrach Delo 12: 107-111.

59. Gudzhedzhiani RB (1973) About copper, zinc, lead, and silver in blood and thyroid tissue of patients with thyrotoxicosis. PhD thesis, Tbilisi medical institute, Tbilisi.

60. Kamenev VF (1963) About trace element contents in thyroid of adults. In: Trace Elements in Agriculture and Medicine. Buryatia publishinghouse, Ulan-Ude, pp. 12-16.

61. Antonova MV, Elinova VG, Voitekhovskaya YaV (1966) Some trace element contents in thyroid and water in endemic goiter region. Zdravookhranenie BSSR 9: 42-44

62. Ianchur NM, Elenevskaia NS, But Gusaim AM, L I Nikhamkin (1967) The content of manganese, aluminum, copper and zinc in the blood and the thyroid gland of patients with goiter. Klin Khir 4: 27-30.

63. Elenevskaia NC, But Gusaim AM, Nikhamkin LP (1968) The content of $\mathrm{Mn}, \mathrm{Al}, \mathrm{Cu}$ и $\mathrm{Zn}$ in thyroid of patients with goiter. In: Proceedings of Vitebsk Medical Institute 12: 123-125.

64. Bredikhin LM, Soroka VP (1969) Trace element metabolism in patients with goiter during therapy. Vrach Delo 51(6): 81-84.

65. Li AA (1971) Cu and Al trace element content in the goitrous transformed thyroids of Kalinin region residents. In: Questions of Thyroid Pathology. Proceedings of Kalinin Medical Institute pp. 27-30.

66. Błazewicz A, Dolliver W, Sivsammye S, Apeksha Deol, Rajinder Randhawa et al. (2010) Determination of cadmium, cobalt, copper, iron, manganese, and zinc in thyroid glands of patients with diagnosed nodular goitre using ion chromatography. J Chromatogr B Analyt Technol Biomed Life Sci 878(1): 34-38. 